\title{
Metamaterials in Medicine: A New Era for Future Orthopedics
}

\author{
Hamid RS Hosseinzadeh ${ }^{1 *}$ and Hamed Hosseinzadeh ${ }^{2}$ \\ ${ }^{1}$ Orthopedic surgeon, USA \\ ${ }^{2}$ Material Science and Engineering, USA
}

*Corresponding author: Hamid RS Hosseinzadeh, Orthopedic surgeon, Associate professor, Rowan University, Glassboro, NJ, USA, Email: hamid@rowan.edu

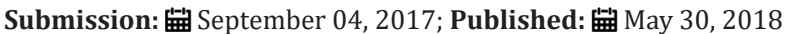

\section{Introduction}

Metamaterials are synthetic materials engineered to provide properties which "may not be available in nature that we know". These materials usually earn their properties from structure rather than composition. The essential property in metamaterials is their unusual and desired properties that appear due to their unique design \& structure [1].

In 1898 J.C. Bose showed the possibility of existence of artificial material by conducting microwave experiment on twisted structure. Later, in 1968, Russian physicist Victor Veselago considered the electromagnetic properties of a hypothetical materialwhose electric permittivity and magnetic permeability were both negative [2]. Such a material, as Veselago mentioned, did not exist in nature. Smith et al. had recognized gradient refractive index medium to bend electromagnetic waves. Metamaterial opened up a new exciting world for the scholars.

The word was first coined by Rodger M Walser (2001) who gave the following definition: Metamaterials are defined as macroscopic composites having a man-made, three dimensional, periodic cellular architecture designed to produce an optimized combination, not available in nature, of two or more responses to a specific excitation [3]. In metamaterials, inclusion of small inhomogeneities can produce effective macroscopic behavior. So, embedding artificially fabricated inclusions in a specific host medium will provide the designer with an ample collection of independent parameters such as properties of host materials and properties related to size, shape, and compositions of inclusions. All these design parameters can play a key role in getting the expected result.

For some years the metamaterials idea has mostly been considered as a means of engineering the electromagnetic response of passive micro- and nanostructured materials. Remarkable results have been achieved in this way: for instance, negative-index media that refract light in the opposite direction from that of conventional materials, chiral materials that rotate the polarization state of light hundreds of thousands of times more strongly than natural optical crystals, and structured thin films with remarkably strong dispersion that can slow light in much the same way as resonant atomic systems with electromagnetically induced transparency [4].

These great achievements in applications of metamaterials, encouraged the biomedical scientists to use these novel materials and their electromagnetic application in medicine. Some of the applications of metamaterials in medicine are described here. Later we describe a new application of the metamaterials in medicine based on another property of them.

\section{Metamaterial antennas}

In Recent years, need for the using of wireless telemetry systems in medicine has significantly increased due to necessity for early diagnosis of diseases and continuous monitoring of physiological parameters. Microwave antennas and sensors are major components of these telemetry systems sincethey provide the communication between the patient and base station. A split-ring resonator (SRR) is one of the metamaterial particles that offer negative permeability [5], which are most commonly used in biomedical sensors [6]. A wireless endoscope, which is a capsule-shaped system used for gastrointestinal monitoring and/ or treatment is one of the most significant applications of the metamaterial antenna.

\section{Metamaterials in cancer detection}

By developing microwave devices and combining it with structures inspired by metamaterials, a very cost-effective device can be developed that can localize an abnormality within the human body with high precision. The basic principle behind the cancer detection is that a minor change in the water content of tissues can produce changes in the permittivity and conductivity values of the tissues. The malignant cells have significantly higher water content than normal tissues. Hence the permittivity and the conductivity of the tumor are different from those ones of a normal tissue at microwave frequencies. The proposed biosensor to detect 
these differences consists of an array of complementary metallic metamaterial resonators [7].

\section{Metamaterials in medical imaging}

A super lens or perfect lens is a lens which uses metamaterials to go beyond the diffraction limit. The diffraction limit is an inherent limitation in conventional optical devises or lenses. In 2000, a type of lens was proposed which consisted of a metamaterial that could compensate for wave decay and reconstructed images in the near field [8]. In 2004, the first superlens with a negative refractive index provided resolution three times better than the diffraction limits and was manufactured at microwave frequencies. In 2005, the first near field superlens which exceeded the diffraction limit was manufactured [9].

\section{Metamaterials in microwave hyperthermia}

Microwaves are used to ablate or evaporate diseased tissue by heating the tissue to a temperature that causes cell death. The generator produces microwave energy which is transmitted through the antennas into the patient. Treatment of cancer by hyperthermia requires applying a carefully controlled dose of heat to the tumor while protecting the surrounding body tissue. The most prominent hallmark of metamaterial lens is the ability of negative-refractive index (NRI) to focus the electromagnetic field of a source [10]. Hence it can generate appropriate focusing spot in the tissue as required in microwave hyperthermia treatment. Recently, conformal microwave array applicators manufactured with low loss left-handed metamaterial lens can be useful for hyperthermia treatment of large tumors. In this method, microwave hyperthermia can be applied by joint heating of the large tumor area with multiple microwave sources [11].

\section{Metamaterials in wireless strain sensing}

RF-microelectromechanical systems (MEMS) strain sensors take advantage of the recent advances in metamaterials. Melik [12] has developed a wireless strain sensor to monitor the healing processes of fractured long bones. The SRR-based sensor displays a characteristic resonance frequency under no load. Hence, by measuring the magnitude of operating frequency shift under an applied load, the progression of fracture healing can be accurately monitored.

\section{Improving the imaging quality of MRI with metamaterials}

A group of researchers from Russia, Australia and the Netherlands has developed a technology that can reduce magnetic resonance imaging (MRI) scanning times by more than $50 \%$, allowing hospitals to remarkably increase the number of scans per day without changing the major parts of their equipment. This extraordinary jump in efficiency is attained by placing a layer of metamaterials onto the bed of the scanner, thereby improving the signal-to-noise ratio.

They have constructed a unique metamaterial resonator in the form of a wire metasurface. The resonance mode of the wire array is spectrally tuned to the nuclear magnetic resonance. This provides a substantial enhancement of the signal-to-noise ratio, thus reducing the MRI scanning time and obtaining images with higher resolutions [13].

\section{Metamaterials as radiation shields (waves AND particles)}

Metamaterials with negative indexes of refraction are certainly interesting and mystifying. The term shielding might be misleading, cloaking or hiding might be a better term. The wavelight or electron-are refracted around the metamaterial objects, as opposed to reflecting (shielding) or absorbing the wave. As such, the metamaterial might protect the underlying structure, but the wave would continue on to hit a more distant structure. This could perhaps serve to shift some of the radiation loads to more distant and robust structures. Thus, these metamaterials can serve to protect the medical personnel from $\mathrm{x}$-rays radiation [14].

\section{The Mechanical metamaterials}

Mechanical properties of a material are governed by the chemical composition and spatial arrangement of constituent elements at multiple length-scales. This essentially limits material properties with respect to each other when selecting materials for specific applications. For example, strength and density are inherently linked, so, in general, the denser the material, the stronger it is in bulk form. Material properties can be controlled via intricate assemblies and structural organization at multiple length-scales as evidenced by naturally occurring cellular materials such as honeycombs, trabecular bone, plant parenchyma, and sponges. It is mainly the architecture of the material's structure at the microand nanoscale, not the chemical composition that governs its mechanical properties [15].

By designing highly ordered architectures in cellular solids, engineers could create the so-called mechanical metamaterials. The ability to decouple properties via micro- and nano-architectural control can allow for unique gathering of, even some opposite, material properties such as ultra-lightweight, low modulus of elasticity, high stiffness and high strength, negative Poisson's Ratio, and negative stiffness. In fact, this approach of choosing a unique property and engineering this material's property via architecture could be described as inverse design engineering. Typically, material properties are considered to be absolute and the created metamaterial is dealt with as a functional structure [15].

Among the interesting properties for a mechanical metamaterial, we have focused on ultra-light weight, low modulus of elasticity, high stiffness and high strength hallmarks of a metamaterial as a building block for the orthopedic devices, esp. endoprostheses. Manufacturing prosthesis from a metamaterial with a low modulus of elasticity near to bone, but at the same time highly stiff and tough, will provide a prosthesis that can behave much like the natural bone in the body. The most significant consequence will be maintaining the normal bone biology around the prosthesis, preventing bone resorption or sclerosis around the prosthesis. Besides, while manufacturing a metamaterial, it is possible to modulate the structure in specific areas to gain a special property in that area. In this way, the prosthesis will have the necessary mechanical properties in junctions and angles, thus, behaving more 
naturally. Metamaterials are usually porous and this porosity can contribute to bone in growth, resulting in tighter bond between prosthesis and host bone without any need for a coating. The last but not the least, when a metamaterial is used to build a prosthesis, wide range of materials could be used like stainless steel, titanium or cobalt and, we can also use some medical grade polymers to manufacture the prosthesis.

Based on all these specifications of metamaterials, we are working on incorporating a natural metamaterial-like structure, the bone, into the structure of a metamaterial, calling it "Bio metamaterial". Bone itself has many hallmarks of a metamaterial, but the symmetrical, repetitive building structure. By turning the bone structure into a repeating, symmetrical structure we hope to make a metamaterial with properties very similar to bone, but much more stiff and tough at the same time. In this way, without a need for coating, we can gain a surface characteristic necessary for the prosthesis, like bacterial resistance, high friction, and low wear, based on the metamaterial structure selected.

There is growing need for engineers and physicists to concentrate and develop equipment specifically suitable for healthcare. Metamaterials is one of the active field of research in the past decadewhich has potential application in healthcare. This paper summarizes the extensive use of metamaterials in medical applications. We hope thatan overview of the mechanical metamaterials and their applications in orthopedics, esp. "bio metamaterials", will open a wide area of research in the future to manufacture a possible permanent prosthesis.

\section{References}

1. Mendhe SE, Kosta YP (2011) Metamaterial properties and applications. International Journal of Information Technology and Knowledge Management 4(1): 85-89.
2. Veselago VG (1968) The electrodynamics of substances with simultaneously negative values of and $\mu$. Sov Phys Usp 10: 509-514.

3. Gangwar K, Paras RPS, Gangwar (2014) Metamaterials: characteristics, process and applications. Advance in Electronic and Electric Engineering 4(1): 97-106.

4. Nikolay I, Zheludev, Kivshar YS (2012) From metamaterials to metadevices. Nature Materials 11(11): 917-924.

5. Pendry JB, Holden AJ, Robbins DJ, Stewart WJ (1999) Magnetism from conductors and enhanced nonlinear phenomena. IEEE Trans on Microwave Theory and Tech 47(11).

6. Kumar H, Kanth RK, Liljeberg P, Tenhunen H (2011) Metamaterial based slotted patch antenna. IEEE $10^{\text {th }}$ International Conference on Telecommunication in Modern Satellite Cable and Broadcasting Services, pp. 43-46

7. Spada L, Bilotti F, Vegni L (2011) Metamaterial biosensor for cancer detection. IEEE Sensors pp. 627-630.

8. Pendry JB (2000) Negative refraction makes a perfect lens. Phys Rev Lett 85(18): 3966-3969.

9. Fang N, Lee H, Sun C, Zhang X (2005) Sub-diffraction-limited optical imaging with a silver superlens. Science 308(5721): 534-537.

10. Wang G, Fang J, Dong X (2007) Resolution of near-field microwave target detection and imaging by using flat LHM lens. IEEE Trans. Antennas Propag 55(12): 3534-3354.

11. Tao Y, Wang G (2012) Hyperthermia of large superficial tumor with a flat LHM lens. IEEE Microwave Symposium Digest, India.

12. Melik R, Unal E, Puttlitz C, Demir HV (2009) Wireless metamaterial RFMEMS strain sensors. Appl Phys Lett 95.

13. Slobozhanyuk AP, Poddubny AN, Raaijmakers AJ, van den Berg CA, Kozachenko AV, et al. (2016) Enhancement of magnetic resonance imaging with Metasurfaces. Adv Mater 28(9): 1832-1838.

14. Zhang S, Genov DA, Sun C, Zhang X (2003) Cloaking of matter waves. Phys Rev Lett 100(12): 123002

15. Gibson JL, Ashby FM (2001) Cellular solids: structure and properties. Cambridge University Press, Cambridge, UK.
Creative Commons Attribution 4.0 International License

For possible submissions Click Here

\section{Submit Article}

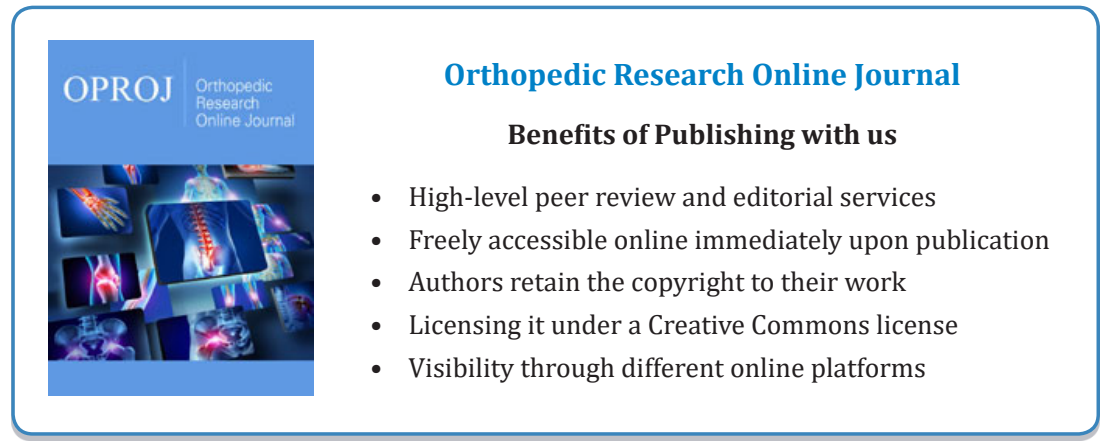

